\title{
Adaptive Modified Function Projective Synchronization between Two Different Hyperchaotic Dynamical Systems
}

\author{
M. M. El-Dessoky, ${ }^{1,2}$ M. T. Yassen, ${ }^{2}$ and E. Saleh ${ }^{2}$ \\ ${ }^{1}$ Department of Mathematics, Faculty of Science, King AbdulAziz University, P.O. Box 80203, \\ Jeddah 21589, Saudi Arabia \\ ${ }^{2}$ Department of Mathematics, Faculty of Science, Mansoura University, Mansoura 35516, Egypt
}

Correspondence should be addressed to M. T. Yassen, mtyassen@yahoo.com

Received 11 November 2011; Accepted 17 December 2011

Academic Editor: Jun-Juh Yan

Copyright (C) 2012 M. M. El-Dessoky et al. This is an open access article distributed under the Creative Commons Attribution License, which permits unrestricted use, distribution, and reproduction in any medium, provided the original work is properly cited.

\begin{abstract}
This work investigates modified function projective synchronization between two different hyperchaotic dynamical systems, namely, hyperchaotic Lorenz system and hyperchaotic Chen system with fully unknown parameters. Based on Lyapunov stability theory, the adaptive control law and the parameter update law are derived to achieve modified function projective synchronized between two diffierent hyperchaotic dynamical systems. Numerical simulations are presented to demonstrate the effectiveness of the proposed adaptive controllers.
\end{abstract}

\section{Introduction}

During the last three decades, synchronization of chaotic systems has attracted increasing attention from scientists and engineers and has been explored intensively both theoretically and experimentally. Since Pecora and Carrol [1] introduced a method to synchronize two identical systems with different initial conditions, many approaches have been proposed for the synchronization of chaotic or hyperchaotic systems such as complete synchronization [1], phase synchronization [2], generalized synchronization [3], lag synchronization [4], intermittent lag synchronization [5], time-scale synchronization [6], intermittent generalized synchronization [7], projective synchronization [8], modified projective synchronization $[9,10]$, and function projective synchronization $[11,12]$. Most of them are based on exactly knowing the system structure and parameters, but in practice, some or all of the system's parameters are unknown. Moreover, these parameters change from time to time. A lot of works have been done to solve this problem using adaptive synchronization [13-16]. Most 
of the methods mentioned above synchronize two identical chaotic systems. Hyperchaotic system is usually classified as a chaotic system with more than one positive Lyapunov exponent, indicating that the chaotic dynamics of the system are expanded in more than one direction giving rise to a more complex attractor. The method of the synchronization of two different hyperchaotic systems is far from being straightforward. There is little work about this challenging problem because it consists of different structures and parameter mismatch of the two hyperchaotic systems. Complete synchronization is characterized by the equality of state variables while evolving in time. Antisynchronization is characterized by the vanishing of the sum of relevant variables. Projective synchronization occurs when the drive and response system could be synchronized up to a scaling factor. Function projective synchronization is the most general definition of projective synchronization. It means that the derive and response systems could be synchronized up to a scaling function.

A focused problem in the study of chaos synchronization is how to design a physically available and simple controller to guarantee the realization of high-quality synchronization in coupled chaotic systems. Linear feedback is of course a practical technique, but the shortcoming is that it needs to find the suitable feedback constant. Recently, Huang proposed a simple adaptive feedback control method, which neednot to estimate or find feedback constant, to effectively synchronize two almost arbitrary identical chaotic systems in his series paper [17-19].

In this work, we investigate modified function projective synchronization (MFPS) between hyperchaotic Lorenz system and hyperchaotic Chen system with fully unknown parameters. This work is organized as follows. In Section 2 the modified function projective synchronization (MFPS) scheme is presented. Section 3 briefly describes hyperchaotic Lorenz system and hyperchaotic Chen system. Section 4 proposes adaptive control laws and parameter update rules for the modified function projective synchronization between hyperchaotic Lorenz system and hyperchaotic Chen system. In Section 5, numerical examples are given to demonstrate the effectiveness of the proposed method. Finally, the conclusions are given in Section 6.

\section{Adaptive Modified Function Projective Synchronization (MFPS) Scheme}

Consider the following master and slave system:

$$
\begin{gathered}
\dot{x}=f(x, t), \\
\dot{y}=g(y, t)+u(x, y, t),
\end{gathered}
$$

where $x, y \in R^{n}$ are the state vector of the system (2.1) and (2.2), respectively; $f, g: R^{n} \rightarrow R^{n}$ are two continuous nonlinear vector functions, $u(x, y, t)$ is the vector controller. We define the error dynamical system as

$$
e(t)=y-M h(t) x,
$$

where $M$ is a constant diagonal matrix $M=\operatorname{diag}\left\{m_{1}, m_{2}, \ldots, m_{n}\right\} \in R^{n \times n}$ and $h(t)$ a continuous differentiable function with $h(t) \neq 0$ for all $t$. 


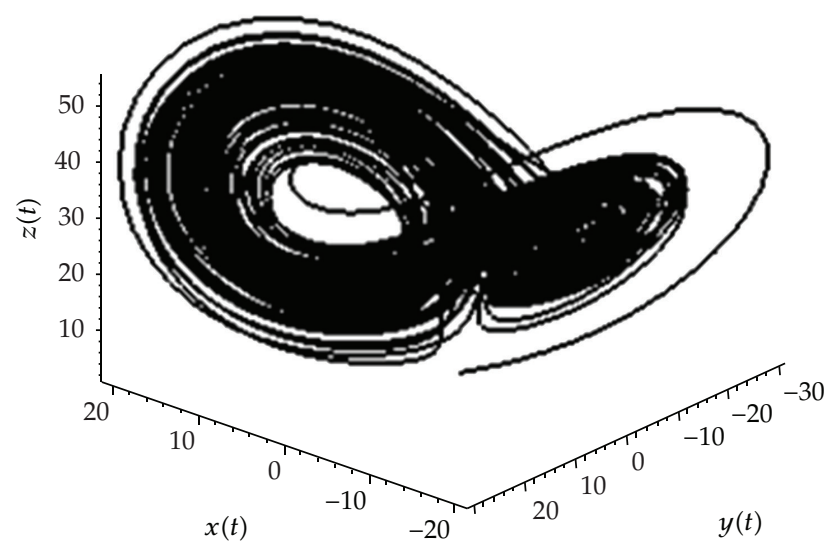

Figure 1: The attractor of hyperchaotic Lorenz dynamical system at $\alpha=10, \beta=28, \gamma=8 / 3$, and $r=0.1$ in $x, y, z$ subspace.

The system (2.1) and (2.2) i said to be in modified function projective synchronization if there exists a constant diagonal matrix $M$ and function $h(t)$, such that $\operatorname{Lim}_{t \rightarrow \infty}\|e(t)\|=0$.

It is easy to see that the definition of modified function projective synchronization encompasses function projective synchronization when the scaling matrix $M$ equals $I$.

\section{System Description}

The hyperchaotic Lorenz system is described as follows [20-22]:

$$
\begin{gathered}
\dot{x}=\alpha(y-x), \\
\dot{y}=\beta x+y-x z-w, \\
\dot{z}=x y-b z, \\
\dot{w}=r y z,
\end{gathered}
$$

where $x, y, z$, and $w$ are state variables and $\alpha, \beta, \gamma$, and $r$ are real constant parameters. In $[21,22]$, it has been shown that the system (3.1) has two positive Lyapunov exponents when $\alpha=10, \beta=28, \gamma=8 / 3$, and $r=0.1$, the system (3.1) exhibits hyperchaotic behavior, see Figure 1 .

Hyperchaotic Chen system is described as [23, 24]

$$
\begin{gathered}
\dot{x}=a(y-x)+w, \\
\dot{y}=d x+c y-x z, \\
\dot{z}=x y-b z, \\
\dot{w}=l w+y z,
\end{gathered}
$$




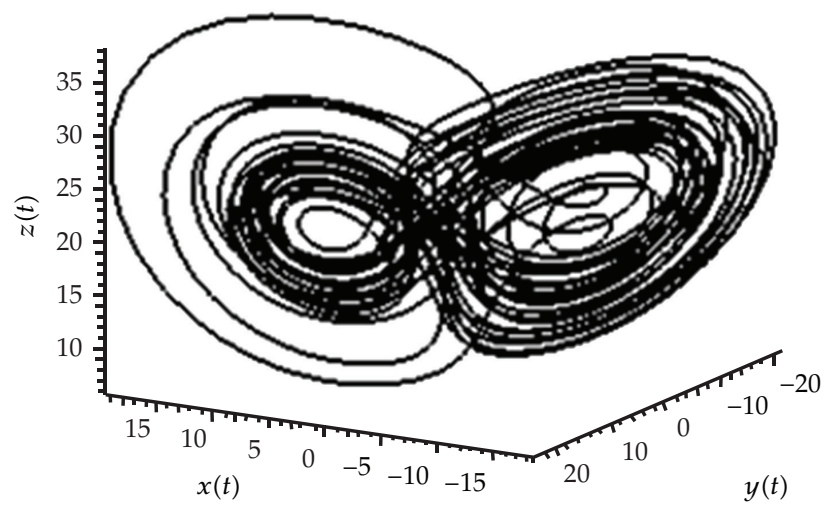

Figure 2: The attractor of hyperchaotic Chen dynamical system at $a=35, b=3, c=12, d=7$, and $l=0.5$ in $x, y, z$ subspace.

where $x, y, z$ and $w$ are state variables and $a, b, c, d$ and $h$ are real constant parameters. When $a=35, b=3, c=12, d=7,0.798 \leq l \leq 0.9$, system (3.2) is periodic, when $a=35, b=3, c=$ $12, d=7,0 \leq l \leq 0.085$, system (3.2) is chaotic; when $a=35, b=3, c=12, d=7,0.085 \leq l \leq$ 0.798 , system (3.2) exhibits hyperchaotic behavior see Figure 2.

\section{Adaptive MFPS between Hyperchaotic Lorenz System and Chen System}

In order to achieve the synchronization behavior between hyperchaotic Lorenz system and hyperchaotic Chen system, we assume that hyperchaotic Lorenz system is the drive system whose four variables are denoted by subscript 1 and hyperchaotic Chen system is the response system whose variables are denoted by subscript 2 . The drive and response systems are described by the following equations, respectively,

$$
\begin{gathered}
\dot{x}_{1}=\alpha\left(y_{1}-x_{1}\right), \\
\dot{y}_{1}=\beta x_{1}+y_{1}-x_{1} z_{1}-w_{1}, \\
\dot{z}_{1}=x_{1} y_{1}-r z_{1}, \\
\dot{w}_{1}=r y_{1} z_{1}, \\
\dot{x}_{2}=a\left(y_{2}-x_{2}\right)+w_{2}+u_{1}, \\
\dot{y}_{2}=d x_{2}+c y_{2}-x_{2} z_{2}+u_{2}, \\
\dot{z}_{2}=x_{2} y_{2}-b z_{2}+u_{3}, \\
\dot{w}_{2}=l w_{2}+y_{2} z_{2}+u_{4},
\end{gathered}
$$

where $U=\left[u_{1}, u_{2}, u_{3}, u_{4}\right]^{T}$ is the nonlinear controller functions which are to be determined later. The term "synchronization," in general, means that the signal effect upon the synchronized system is very small in comparison with the amplitude of proper oscillations of the 
system but, nevertheless, it is enough to change the system behavior and to "impose" the rhythm of external influence to it. The two hyperchaotic dynamical systems can be synchronized in the sense that

$$
\begin{aligned}
& \lim _{t \rightarrow \infty}\left|x_{2}-m_{1} h(t) x_{1}\right|=0 \\
& \lim _{t \rightarrow \infty}\left|y_{2}-m_{2} h(t) y_{1}\right|=0 \\
& \lim _{t \rightarrow \infty}\left|z_{2}-m_{3} h(t) z_{1}\right|=0 \\
& \lim _{t \rightarrow \infty}\left|w_{2}-m_{4} h(t) w_{1}\right|=0
\end{aligned}
$$

where $m_{i},(i=1,2,3,4)$ is the scaling factor and $h(t)$ the scaling function.

We have the following error dynamical system:

$$
\begin{aligned}
& \dot{e}_{x}=\dot{x}_{2}-m_{1} h(t) \dot{x}_{1}-m_{1} \dot{h}(t) x_{1}, \\
& \dot{e}_{y}=\dot{y}_{2}-m_{2} h(t) \dot{y}_{1}-m_{2} \dot{h}(t) y_{1}, \\
& \dot{e}_{z}=\dot{z}_{2}-m_{3} h(t) \dot{z}_{1}-m_{3} \dot{h}(t) z_{1}, \\
& \dot{e}_{w}=\dot{w}_{2}-m_{4} h(t) \dot{w}_{1}-m_{4} \dot{h}(t) w_{1},
\end{aligned}
$$

where $e_{x}=x_{2}-m_{1} h(t) x_{1}, e_{y}=y_{2}-m_{1} h(t) y_{1}, e_{z}=z_{2}-m_{1} h(t) z_{1}$, and $e_{w}=w_{2}-m_{1} h(t) w_{1}$.

Substitution of (4.1) and (4.2) in (4.4) yields following error dynamical system

$$
\begin{gathered}
\dot{e}_{x}=a\left(y_{2}-x_{2}\right)+w_{2}+u_{1}-m_{1} h(t) \alpha\left(y_{1}-x_{1}\right)-m_{1} \dot{h}(t) x_{1} \\
\dot{e}_{y}=d x_{2}+c y_{2}-x_{2} z_{2}+u_{2}-m_{2} h(t)\left(\beta x_{1}+y_{1}-x_{1} z_{1}-w_{1}\right)-m_{2} \dot{h}(t) y_{1} \\
\dot{e}_{z}=x_{2} y_{2}-b z_{2}+u_{3}-m_{3} h(t)\left(x_{1} y_{1}-\gamma z_{1}\right)-m_{3} \dot{h}(t) z_{1} \\
\dot{e}_{w}=l w_{2}+y_{2} z_{2}+u_{4}-m_{4} h(t) r y_{1} z_{1}-m_{4} \dot{h}(t) w_{1} .
\end{gathered}
$$

Our aim is to find control laws $u_{i}(i=1,2,3,4)$ for stabilizing the error variables of the system at the origin. For this end, we propose following control law:

$$
\begin{gathered}
u_{1}=m_{1} h(t) \alpha_{1}\left(y_{1}-x_{1}\right)+m_{1} \dot{h}(t) x_{1}-a_{1}\left(x_{2}-y_{2}\right)-w_{2}-k_{1} e_{x} \\
u_{2}=m_{2} h(t)\left(y_{1}+\beta_{1} x_{1}-w_{1}-x_{1} z_{1}\right)+x_{2} z_{2}+m_{2} \dot{h}(t) y_{1}-d_{1} x_{2}-c_{1} y_{2}-k_{2} e_{y}, \\
u_{3}=m_{3} h(t)\left(x_{1} y_{1}-\gamma_{1} z_{1}\right)+m_{3} \dot{h}(t) z_{1}+b_{1} z_{2}-x_{2} y_{2}-k_{3} e_{z \prime} \\
u_{4}=m_{4} h(t) r_{1} y_{1} z_{1}+m_{4} \dot{h}(t) w_{1}-l_{1} w_{2}-y_{2} z_{2}-k_{4} e_{w},
\end{gathered}
$$


and the update laws for the unknown parameters $\alpha_{1}, \beta_{1}, \gamma_{1}, r_{1}, a_{1}, b_{1}, c_{1}, d_{1}$, and $l_{1}$ are

$$
\begin{gathered}
\dot{\alpha}_{1}=\left(x_{1}-y_{1}\right) m_{1} h(t) e_{x}-k_{5}\left(\alpha_{1}-\alpha\right), \\
\dot{\beta}_{1}=-x_{1} m_{2} h(t) e_{y}-k_{6}\left(\beta_{1}-\beta\right), \\
\dot{\gamma}_{1}=z_{1} m_{3} h(t) e_{z}-k_{7}\left(\gamma_{1}-\gamma\right), \\
\dot{r}_{1}=-y_{1} z_{1} m_{4} h(t) e_{w}-k_{8}\left(r_{1}-r\right), \\
\dot{a}_{1}=\left(y_{2}-x_{2}\right) e_{x}-k_{9}\left(a_{1}-a\right), \\
\dot{b}_{1}=-z_{2} e_{z}-k_{10}\left(b_{1}-b\right), \\
\dot{c}_{1}=y_{2} e_{y}-k_{11}\left(c_{1}-c\right), \\
\dot{d}_{1}=x_{2} e_{y}-k_{12}\left(d_{1}-d\right), \\
\dot{l}_{1}=w_{2} e_{w}-k_{13}\left(l_{1}-l\right),
\end{gathered}
$$

where $k_{i}>0(i=1,2,3, \ldots, 13)$.

Theorem 4.1. For given constant scaling matrix $M$ and scaling function $h(t)$, the MFPS between two systems (4.1) and (4.2) will occur by the control law (4.6) and update law (4.7), and satisfy

$$
\begin{aligned}
\lim _{t \rightarrow \infty}\left|\alpha_{1}-\alpha\right| & =\lim _{t \rightarrow \infty}\left|\beta_{1}-\beta\right|=\lim _{t \rightarrow \infty}\left|\gamma_{1}-\gamma\right|=\lim _{t \rightarrow \infty}\left|r_{1}-r\right|=\lim _{t \rightarrow \infty}\left|a_{1}-a\right| \\
& =\lim _{t \rightarrow \infty}\left|b_{1}-b\right|=\lim _{t \rightarrow \infty}\left|c_{1}-c\right|=\lim _{t \rightarrow \infty}\left|d_{1}-d\right|=\lim _{t \rightarrow \infty}\left|l_{1}-l\right|=0 .
\end{aligned}
$$

Proof. Define a Lyapunov function,

$$
V(e)=\frac{1}{2}\left(e_{x}^{2}+e_{y}^{2}+e_{z}^{2}+e_{w}^{2}+e_{\alpha}^{2}+e_{\beta}^{2}+e_{\gamma}^{2}+e_{r}^{2}+e_{a}^{2}+e_{b}^{2}+e_{c}^{2}+e_{d}^{2}+e_{l}^{2}\right)
$$

where

$$
\begin{gathered}
e_{\alpha}=\alpha_{1}-\alpha, \quad e_{\beta}=\beta_{1}-\beta, \quad e_{\gamma}=\gamma_{1}-\gamma, \quad e_{r}=r_{1}-r, \quad e_{a}=a_{1}-a, \\
e_{b}=b_{1}-b, \quad e_{c}=c_{1}-c, \quad e_{d}=d_{1}-d, \quad e_{l}=l_{1}-l .
\end{gathered}
$$

The time derivative of the Lyapunov function along the trajectory of error system (4.9) is

$$
\begin{aligned}
\frac{d V(e)}{d t}= & e_{x} \dot{e}_{x}+e_{y} \dot{e}_{y}+e_{z} \dot{e}_{z}+e_{w} \dot{e}_{w}+e_{\alpha} \dot{e}_{\alpha}+e_{\beta} \dot{e}_{\beta}+e_{\gamma} \dot{e}_{\gamma} \\
& +e_{r} \dot{e}_{r}+e_{a} \dot{e}_{a}+e_{b} \dot{e}_{b}+e_{c} \dot{e}_{c}+e_{d} \dot{e}_{d}+e_{l} \dot{e}_{l} \\
= & e_{x} \dot{e}_{x}+e_{y} \dot{e}_{y}+e_{z} \dot{e}_{z}+e_{w} \dot{e}_{w}+e_{\alpha} \dot{\alpha}_{1}+e_{\beta} \dot{\beta}_{1}+e_{\gamma} \dot{\gamma}_{1} \\
& +e_{r} \dot{r}_{1}+e_{a} \dot{a}_{1}+e_{b} \dot{b}_{1}+e_{c} \dot{c}_{1}+e_{d} \dot{d}_{1}+e_{l} \dot{l}_{1} .
\end{aligned}
$$


Inserting (4.6) and (4.7) into (4.11) yields the following:

$$
\begin{aligned}
\frac{d V(e)}{d t}= & -k_{1} e_{x}^{2}-k_{2} e_{y}^{2}-k_{3} e_{z}^{2}-k_{4} e_{w}^{2}-k_{5} e_{\alpha}^{2}-k_{6} e_{\beta}^{2}-k_{7} e_{\gamma}^{2}-k_{8} e_{r}^{2} \\
& -k_{9} e_{a}^{2}-k_{10} e_{b}^{2}-k_{11} e_{c}^{2}-k_{12} e_{d}^{2}-k_{13} e_{l}^{2}=-K e^{2},
\end{aligned}
$$

where $e=\left(e_{x}, e_{y}, e_{z}, e_{w}, e_{\alpha}, e_{\beta}, e_{\gamma}, e_{r}, e_{a}, e_{b}, e_{c}, e_{d}, e_{l}\right)^{T}$ and $K=\operatorname{diag}\left(k_{1}, k_{2}, k_{3}, k_{4}, k_{5}, k_{6}, k_{7}, k_{8}\right.$, $\left.k_{9}, k_{10}, k_{11}, k_{12}, k_{13}\right)^{T}$.

Since $d V(e) / d t \leq 0$, we have $e_{x}, e_{y}, e_{z}, e_{w}, e_{\alpha}, e_{\beta}, e_{\gamma}, e_{r}, e_{a}, e_{b}, e_{c}, e_{d}, e_{l} \rightarrow 0$ as $t \rightarrow \infty$, $\lim _{t \rightarrow \infty}\|e\|=0$. of MFPS.

Therefore, the drive system (4.1) synchronizes the response system (4.2) in the sense

Remark 4.2. Note that complete synchronization and antisynchronization between two different hyperchaotic dynamical systems are special cases of MFPS with the scaling function $h(t)=1$ and the scaling factors $m_{i}=1$ and $m_{i}=-1(i=1,2,3,4)$, respectively.

Remark 4.3. Note that function projective synchronization (FPS) between two different hyperchaotic dynamical systems is special case of MFPS with the scaling factors $m_{i}=1(i=1,2$, $3,4)$, and the scaling function $h(t)$ is chosen later.

Remark 4.4. Note that generalized projective synchronization (GPS) and modified generalized projective synchronization (MGPS) between two different hyperchaotic dynamical systems are special cases of MFPS with the scaling function $h(t)=1$ and the scaling factors $m_{i}$ are equal and $m_{i}$ are not equal $(i=1,2,3,4)$, respectively.

By suitable choosing for $h(t)$, we can achieve modified function projective synchronization, complete synchronization, antisynchronization, function projective synchronization, generalized projective synchronization, modified generalized projective synchronization, between two different hyperchaotic systems (see Examples 5.1-5.6).

\section{Numerical Results}

In this section, numerical examples are used to demonstrate the effectiveness of the proposed method. By using Maple 12 to solve the systems of differential equations (4.1), (4.2), (4.6), and (4.7), we assume that the initial conditions of the drive system are $x_{1}(0)=2, y_{1}(0)=2, z_{1}(0)=$ 3 , and $w_{1}(0)=1$, and the initial conditions of the response system are $x_{2}(0)=6, y_{2}(0)=5$, $z_{2}(0)=3$, and $w_{2}(0)=3$. The initial conditions of the estimated parameters are chosen as $\alpha_{1}(0)=0, \beta_{1}(0)=0, \gamma_{1}(0)=0, r_{1}(0)=0, a_{1}(0)=0, b_{1}(0)=0, c_{1}(0)=0, d_{1}(0)=0$ and $l_{1}(0)=0$.

Let the scaling function be $h(t)=\sin (0.1 \pi t)$ and the scaling factors are chosen as $m_{1}=$ $2, m_{2}=3, m_{3}=5$, and $m_{4}=0.5$. The simulation of the error dynamical system between hyperchaotic Lorenz system and hyperchaotic Chen system without control functions is shown in Figure 3(a) displays the $e_{x}=x_{2}-m_{1} h(t) x_{1}$, Figure 3(b) displays the $e_{y}=y_{2}-m_{1} h(t) y_{1}$, Figure 3(c) displays the $e_{z}=z_{2}-m_{1} h(t) z_{1}$, and Figure 3(d) displays the $e_{w}=w_{2}-m_{1} h(t) w_{1}$.

Example 5.1. Let the scaling function be $h(t)=\sin (0.1 \pi t)$ and the scaling factors are chosen as $m_{1}=2, m_{2}=3, m_{3}=5$, and $m_{4}=0.5$. Furthermore, the control gains are chosen as $k_{1}=k_{2}=$ $k_{3}=k_{4}=3, k_{5}=k_{6}=k_{7}=k_{8}=k_{9}=k_{10}=k_{11}=k_{12}=h_{13}=2$. Figure 4 displays the MFPS between systems (4.1) and (4.2). Figure 5 show that the estimates $\alpha_{1}(t), \beta_{1}(t), \gamma_{1}(t), r_{1}(t)$ of the unknown parameters converge to $\alpha=10, \beta=28, \gamma=8 / 3$, and $r=0.1$ as $t \rightarrow \infty$. Figure 6 


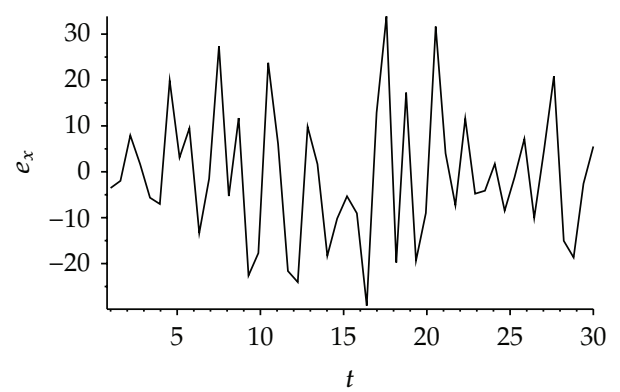

(a)

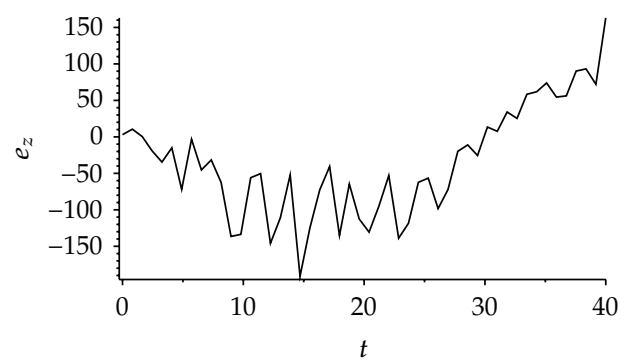

(c)

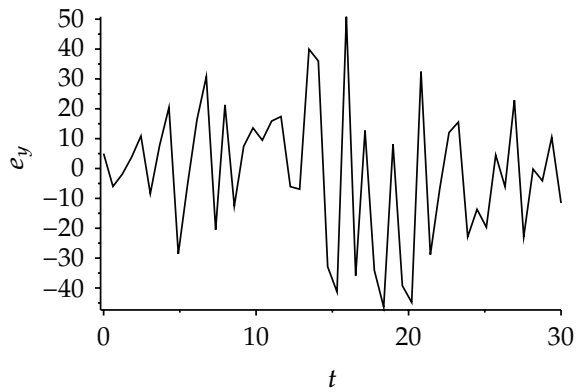

(b)

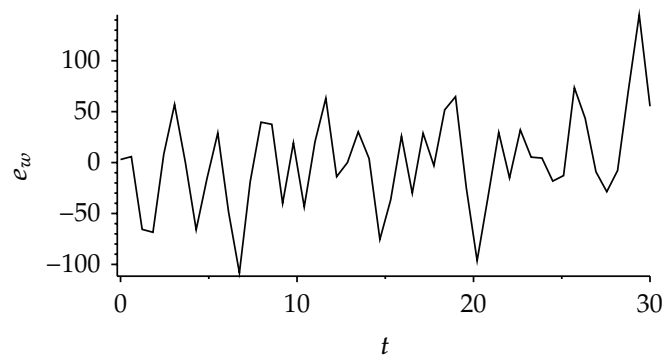

(d)

Figure 3: The behavior of the trajectories $e_{x}, e_{y}, e_{z}$, and $e_{w}$ of the error system between hyperchaotic Lorenz system and hyperchaotic Chen system without control functions.

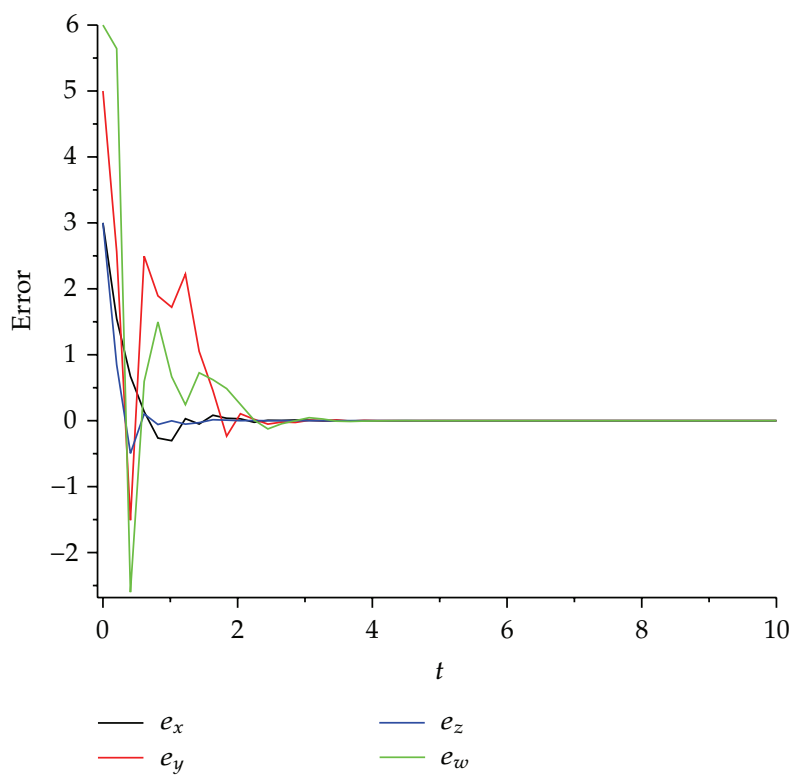

Figure 4: The behavior of the trajectories $e_{x}, e_{y}, e_{z}$, and $e_{w}$ of the error system between hyperchaotic Lorenz system and hyperchaotic Chen system for MFPS. 


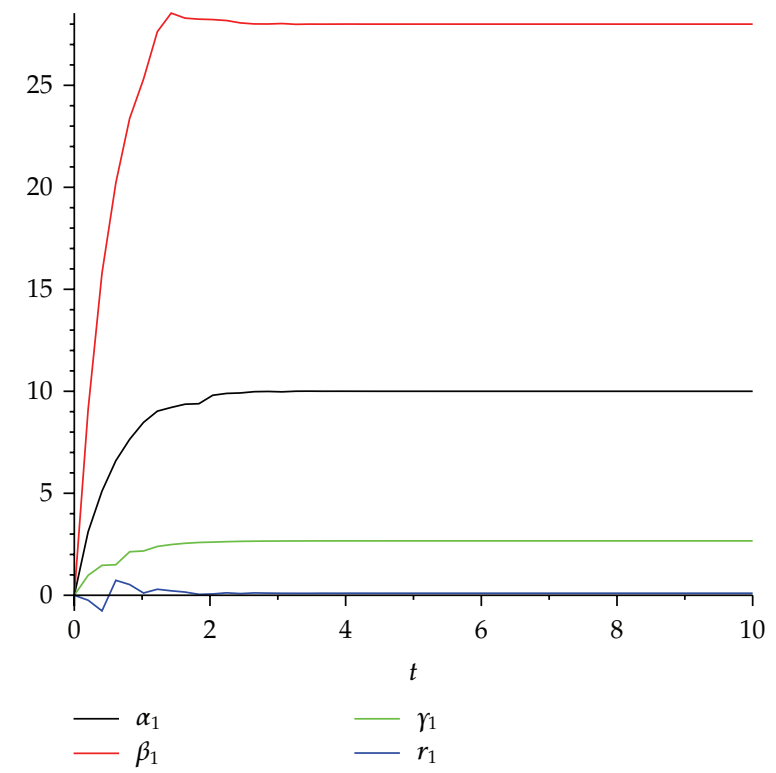

Figure 5: The estimates $\alpha_{1}(t), \beta_{1}(t), \gamma_{1}(t), r_{1}(t)$ of the unknown parameters converges to $\alpha=10, \beta=28, \gamma=$ $8 / 3$, and $r=0.1$ as $t$ tends to 3 .

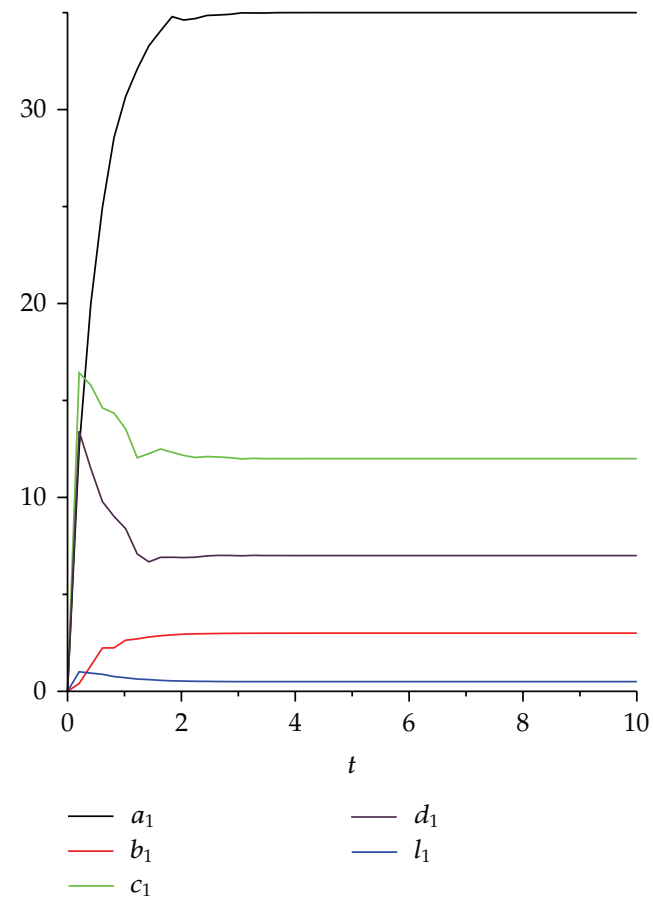

Figure 6: The estimates $a_{1}(t), b_{1}(t), c_{1}(t), d_{1}(t), l_{1}(t)$ of the unknown parameters converge to $a=35, b=$ $3, c=12, d=7$, and $l=0.5$ as $t$ tends to 3 . 


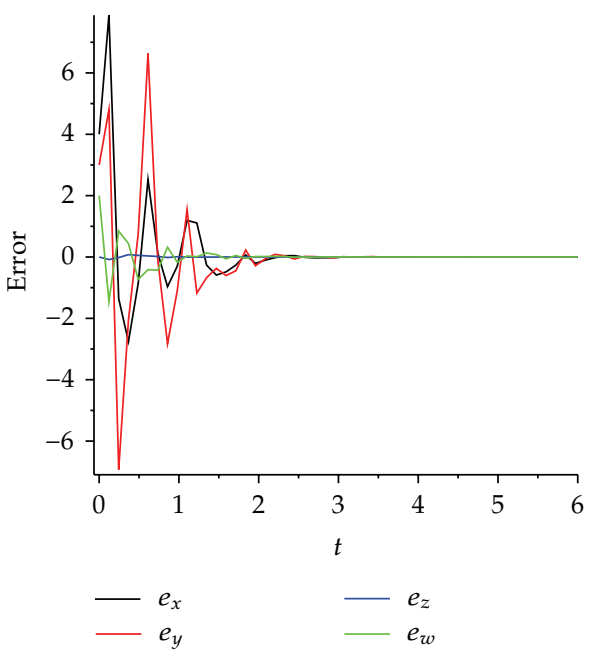

(a)

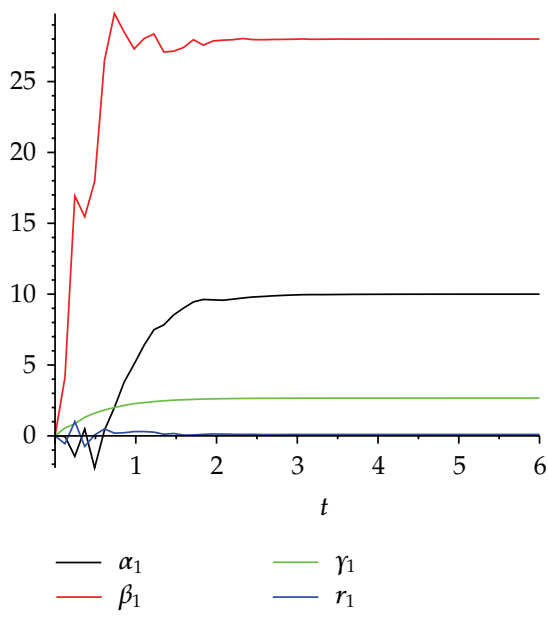

(b)

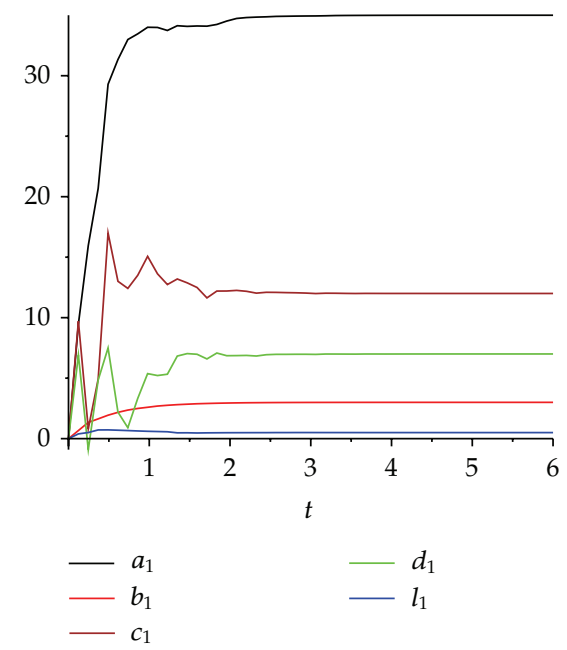

(c)

Figure 7: (a) The behavior of the trajectories $e_{x}, e_{y}, e_{z}$, and $e_{w}$ of the error system between hyperchaotic Lorenz system and hyperchaotic Chen system for complete synchronization. (b) the estimates $\alpha_{1}(t), \beta_{1}(t)$, $\gamma_{1}(t), r_{1}(t)$ of the unknown parameters converge to $\alpha=10, \beta=28, \gamma=8 / 3$, and $r=0.1$ as $t$ tends to 3 . (c) the estimates $a_{1}(t), b_{1}(t), c_{1}(t), d_{1}(t), l_{1}(t)$ of the unknown parameters converges to $a=35, b=3, c=$ $12, d=7$, and $l=0.5$ as $t$ tends to 3 .

show that the estimates $a_{1}(t), b_{1}(t), c_{1}(t), d_{1}(t), l_{1}(t)$ of the unknown parameters converge to $a=35, b=3, c=12, d=7$, and $l=0.5$ as $t \rightarrow \infty$.

Example 5.2. Let the scaling function be $h(t)=1$ and the scaling factors are chosen as $m_{1}=m_{2}=m_{3}=m_{4}=1$. Furthermore, the control gains are chosen as $k_{1}=k_{2}=k_{3}=k_{4}=3$, $k_{5}=k_{6}=k_{7}=k_{8}=k_{9}=k_{10}=k_{11}=k_{12}=h_{13}=2$. Figure 7(a) displays the complete synchronization between systems (4.1) and (4.2). Figure 7(b) show that the estimates $\alpha_{1}(t)$, $\beta_{1}(t), \gamma_{1}(t), r_{1}(t)$ of the unknown parameters converge to $\alpha=10, \beta=28, \gamma=8 / 3$, and $r=0.1$ as $t \rightarrow \infty$. Figure 7(c) show that the estimates $a_{1}(t), b_{1}(t), c_{1}(t), d_{1}(t), l_{1}(t)$ of the unknown parameters converge to $a=35, b=3, c=12, d=7$, and $l=0.5$ as $t \rightarrow \infty$. 


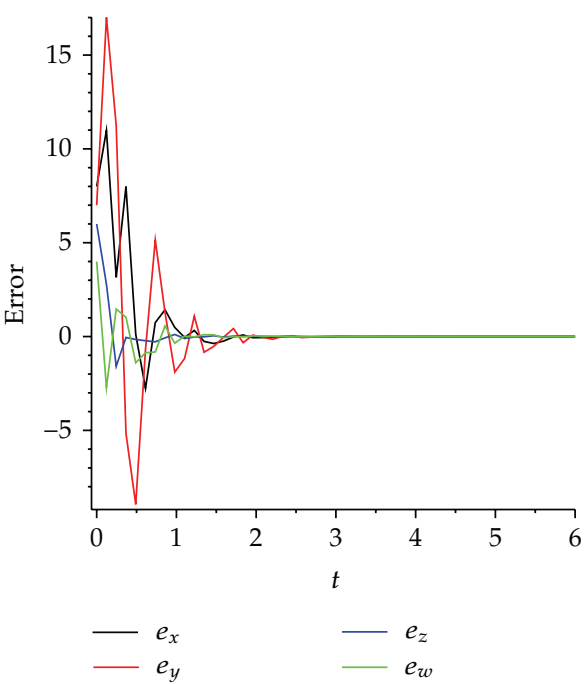

(a)

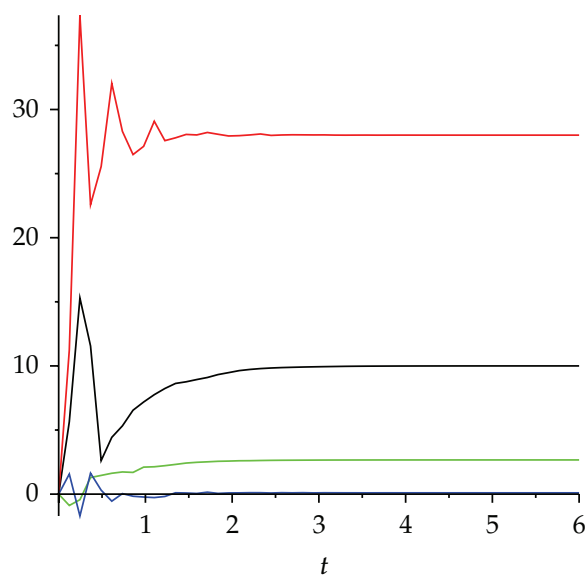

$\alpha_{1}$
$-\beta_{1}$

$\gamma_{1}$

(b)

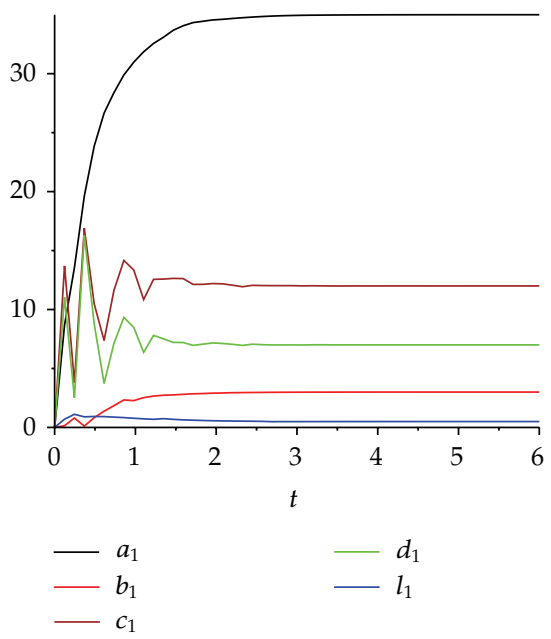

(c)

Figure 8: (a) The behaviour of the trajectories $e_{x}, e_{y}, e_{z}$ and $e_{w}$ of the error system between hyperchaotic Lorenz system and hyperchaotic Chen system for anti-synchronization. (b): Show the estimates $\alpha_{1}(t), \beta_{1}(t)$, $\gamma_{1}(t), r_{1}(t)$ of the unknown parameters converges to $\alpha=10, \beta=28, \gamma=8 / 3$ and $r=0.1$ as $t$ tends to 3 . (c): Show the estimates $a_{1}(t), b_{1}(t), c_{1}(t), d_{1}(t), l_{1}(t)$ of the unknown parameters converges to $a=35, b=$ $3, c=12, d=7$ and $l=0.5$ as $t$ tends to 3 .

Example 5.3. Let the scaling function be $h(t)=1$ and the scaling factors are chosen as $m_{1}=$ $m_{2}=m_{3}=m_{4}=-1$. Furthermore, the control gains are chosen as $k_{1}=k_{2}=k_{3}=k_{4}=3, k_{5}=$ $k_{6}=k_{7}=k_{8}=k_{9}=k_{10}=k_{11}=k_{12}=h_{13}=2$. Figure 8(a) displays the anti-synchronization between systems (4.1) and (4.2). Figure 8(b) shows the estimates $\alpha_{1}(t), \beta_{1}(t), \gamma_{1}(t), r_{1}(t)$ of the unknown parameters converge to $\alpha=10, \beta=28, \gamma=8 / 3$, and $r=0.1$ as $t \rightarrow \infty$. Figure 8(c) shows the estimates $a_{1}(t), b_{1}(t), c_{1}(t), d_{1}(t), l_{1}(t)$ of the unknown parameters converge to $a=35, b=3, c=12, d=7$, and $l=0.5$ as $t \rightarrow \infty$. 


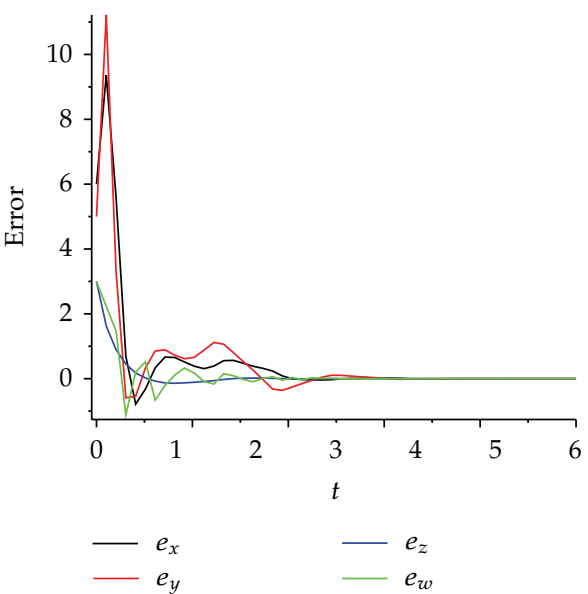

(a)

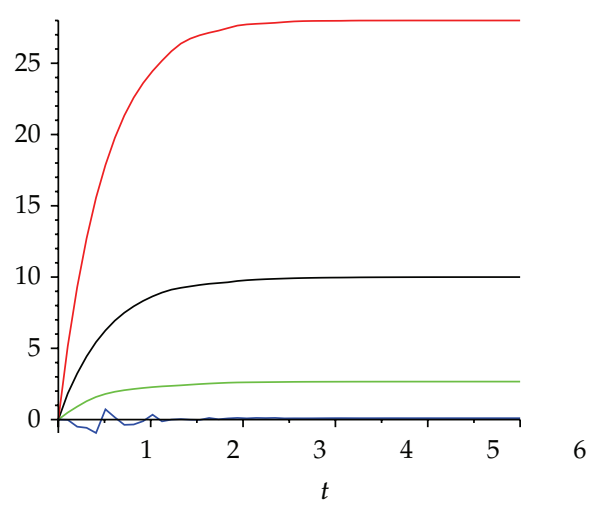

$\begin{array}{ll}\alpha_{1} \\ \beta_{1}\end{array} \quad-r_{1}$

(b)

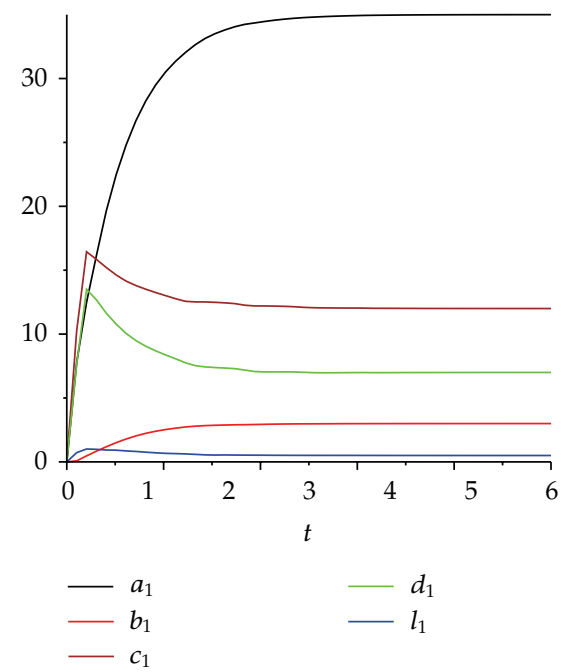

(c)

Figure 9: (a) The behavior of the trajectories $e_{x}, e_{y}, e_{z}$, and $e_{w}$ of the error system between hyperchaotic Lorenz system and hyperchaotic Chen system for FPS. (b) the estimates $\alpha_{1}(t), \beta_{1}(t), \gamma_{1}(t), r_{1}(t)$ of the unknown parameters converge to $\alpha=10, \beta=28, \gamma=8 / 3$, and $r=0.1$ as $t$ tends to 3 . (c) the estimates $a_{1}(t), b_{1}(t), c_{1}(t), d_{1}(t), l_{1}(t)$ of the unknown parameters converge to $a=35, b=3, c=12, d=7$, and $l=0.5$ as $t$ tends to 3 .

Example 5.4. Let the scaling function be $h(t)=\sin (0.1 \pi t)$ and the scaling factors are chosen as $m_{1}=m_{2}=m_{3}=m_{4}=1$. Furthermore, the control gains are chosen as $k_{1}=k_{2}=k_{3}=$ $k_{4}=3, k_{5}=k_{6}=k_{7}=k_{8}=k_{9}=k_{10}=k_{11}=k_{12}=h_{13}=2$. Figure 9(a) displays the FPS between systems (4.1) and (4.2). Figure 9(b) shows the estimates $\alpha_{1}(t), \beta_{1}(t), \gamma_{1}(t), r_{1}(t)$ of the unknown parameters converge to $\alpha=10, \beta=28, \gamma=8 / 3$, and $r=0.1$ as $t \rightarrow \infty$. Figure 9(c) shows the estimates $a_{1}(t), b_{1}(t), c_{1}(t), d_{1}(t), l_{1}(t)$ of the unknown parameters converge to $a=35, b=3, c=12, d=7$, and $l=0.5$ as $t \rightarrow \infty$. 


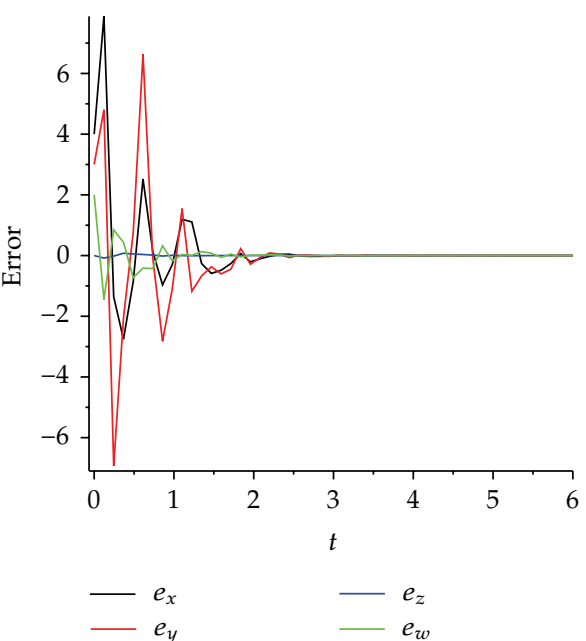

(a)

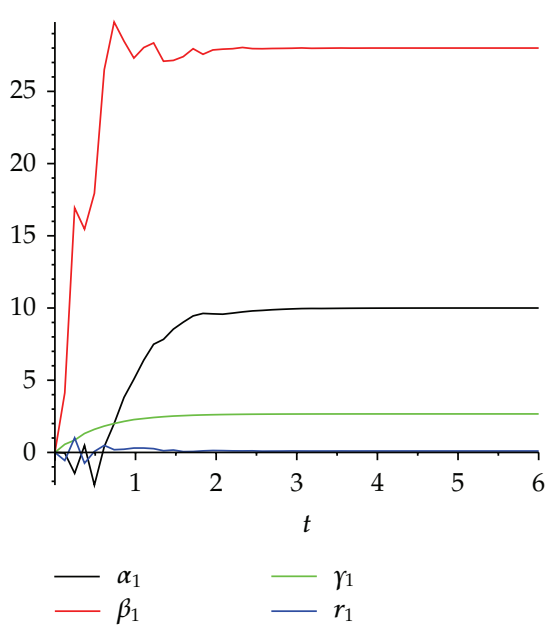

(b)

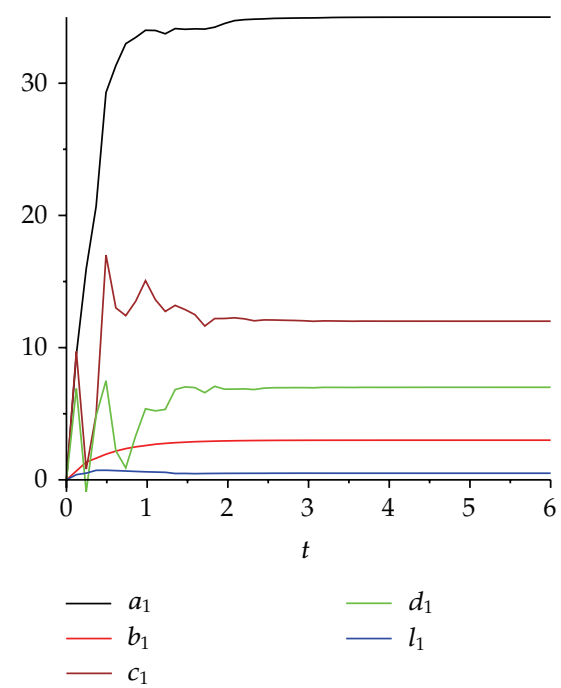

(c)

Figure 10: (a) The behavior of the trajectories $e_{x}, e_{y}, e_{z}$, and $e_{w}$ of the error system between hyperchaotic Lorenz system and hyperchaotic Chen system for GPS. (b) the estimates $\alpha_{1}(t), \beta_{1}(t), \gamma_{1}(t), r_{1}(t)$ of the unknown parameters converges to $\alpha=10, \beta=28, \gamma=8 / 3$, and $r=0.1$ as $t$ tends to 3 . (c) the estimates $a_{1}(t), b_{1}(t), c_{1}(t), d_{1}(t), l_{1}(t)$ of the unknown parameters converges to $a=35, b=3, c=12, d=7$, and $l=0.5$ as $t$ tends to 3 .

Example 5.5. Let the scaling function be $h(t)=1$ and the scaling factors are chosen as $m_{1}=$ $m_{2}=m_{3}=m_{4}=0.5$. Furthermore, the control gains are chosen as $k_{1}=k_{2}=k_{3}=k_{4}=3, k_{5}=$ $k_{6}=k_{7}=k_{8}=k_{9}=k_{10}=k_{11}=k_{12}=h_{13}=2$. Figure 10(a) displays the GPS between systems (4.1) and (4.2). Figure 10(b) shows the estimates $\alpha_{1}(t), \beta_{1}(t), \gamma_{1}(t), r_{1}(t)$ of the unknown parameters converge to $\alpha=10, \beta=28, \gamma=8 / 3$, and $r=0.1$ as $t \rightarrow \infty$. Figure 10(c) shows the estimates $a_{1}(t), b_{1}(t), c_{1}(t), d_{1}(t), l_{1}(t)$ of the unknown parameters converges to $a=35, b=3, c=12, d=7$, and $l=0.5$ as $t \rightarrow \infty$. 


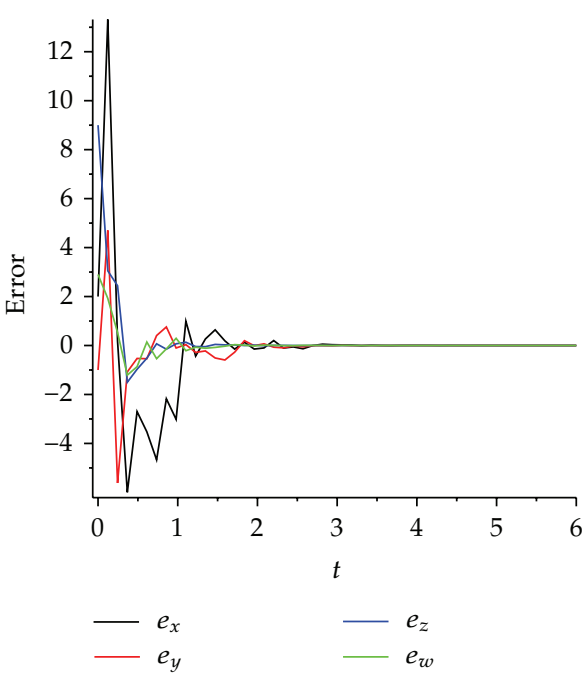

(a)

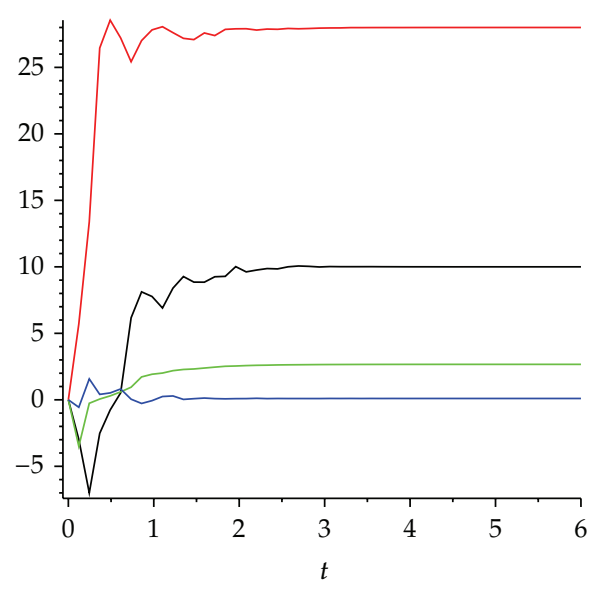

$\begin{array}{ll}\alpha_{1} & -\gamma_{1} \\ \beta_{1} & -r_{1}\end{array}$

(b)

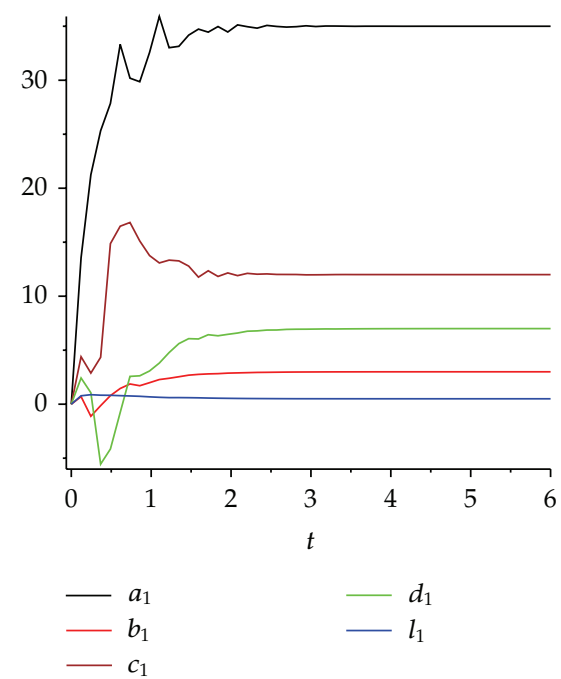

(c)

Figure 11: (a) The behaviour of the trajectories $e_{x}, e_{y}, e_{z}$, and $e_{w}$ of the error system between hyperchaotic Lorenz system and hyperchaotic Chen system for MGPS. (b) the estimates $\alpha_{1}(t), \beta_{1}(t), \gamma_{1}(t), r_{1}(t)$ of the unknown parameters converge to $\alpha=10, \beta=28, \gamma=8 / 3$, and $r=0.1$ as $t$ tends to 3 . (c) the estimates $a_{1}(t), b_{1}(t), c_{1}(t), d_{1}(t), l_{1}(t)$ of the unknown parameters converge to $a=35, b=3, c=12, d=7$, and $l=$ 0.5 as $t$ tends to 3 .

Example 5.6. Let the scaling function be $h(t)=1$ and the scaling factors are chosen as $m_{1}=$ $2, m_{2}=3, m_{3}=-2$, and $m_{4}=0.1$. Furthermore, the control gains are chosen as $k_{1}=k_{2}=k_{3}=$ $k_{4}=3, k_{5}=k_{6}=k_{7}=k_{8}=k_{9}=k_{10}=k_{11}=k_{12}=h_{13}=2$. Figure 11(a) displays the MGPS between systems (4.1) and (4.2). Figure 11(b) show that the estimates $\alpha_{1}(t), \beta_{1}(t), \gamma_{1}(t), r_{1}(t)$ of the unknown parameters converge to $\alpha=10, \beta=28, \gamma=8 / 3$, and $r=0.1$ as $t \rightarrow \infty$. Figure $11(\mathrm{c})$ show that the estimates $a_{1}(t), b_{1}(t), c_{1}(t), d_{1}(t), l_{1}(t)$ of the unknown parameters converge to $a=35, b=3, c=12, d=7$, and $l=0.5$ as $t \rightarrow \infty$. 


\section{Conclusions}

This work investigated modified function projective synchronization between the hyperchaotic Lorenz system and hyperchaotic Chen system with fully unknown parameters. Based on Lyapunov stability theory, we design adaptive synchronization controllers ${ }^{\sim}$ with corresponding parameter update laws to synchronize the two systems. The MFPS includes complete synchronization, antisynchronization, function projective synchronization (FPS), generalized projective synchronization (GPS), and modified generalized projective synchronization (MGPS). All the theoretical results are verified by numerical simulations to demonstrate the effectiveness of the proposed synchronization schemes. Thus, our synchronization method is successful for some systems with two positive Lyapunov exponents.

\section{Acknowledgment}

The authors would like to thank the editor and the anonymous reviewers for their constructive comments and suggestions to improve the quality of the paper. The first author acknowledges with thanks the Deanship of Scientific Research (DSR), King Abdulaziz University, Jeddah, Saudi Arabia for his support this article.

\section{References}

[1] L. M. Pecora and T. L. Carroll, "Synchronization in chaotic systems," Physical Review Letters, vol. 64, no. 8, pp. 821-824, 1990.

[2] M. G. Rosenblum, A. S. Pikovsky, and J. Kurths, "Phase synchronization of chaotic oscillators," Physical Review Letters, vol. 76, no. 11, pp. 1804-1807, 1996.

[3] N. F. Rulkov, M. M. Sushchik, L. S. Tsimring, and H. D. I. Abarbanel, "Generalized synchronization of chaos in directionally coupled chaotic systems," Physical Review E, vol. 51, no. 2, pp. 980-994, 1995.

[4] M. G. Rosenblum, A. S. Pikovsky, and J. Kurths, "From phase to lag synchronization in coupled chaotic oscillators," Physical Review Letters, vol. 78, no. 22, pp. 4193-4196, 1997.

[5] S. Boccaletti and D. L. Valladares, "Characterization of intermittent lag synchronization," Physical Review E, vol. 62, no. 5 B, pp. 7497-7500, 2000.

[6] A. E. Hramov and A. A. Koronovskii, "An approach to chaotic synchronization," Chaos, vol. 14, no. 3 , pp. 603-610, 2004.

[7] A. E. Hramov, A. A. Koronovskii, and O. I. Moskalenko, "Generalized synchronization onset," Europhysics Letters, vol. 72, no. 6, pp. 901-907, 2005.

[8] R. Mainieri and J. Rehacek, "Projective synchronization in three-dimensional chaotic systems," Physical Review Letters, vol. 82, no. 15, pp. 3042-3045, 1999.

[9] G. H. Li, "Generalized projective synchronization between Lorenz system and Chen's system," Chaos, Solitons and Fractals, vol. 32, no. 4, pp. 1454-1458, 2007.

[10] G.-H. Li, "Modified projective synchronization of chaotic system," Chaos, Solitons and Fractals, vol. 32, no. 5, pp. 1786-1790, 2007.

[11] L. Runzi, "Adaptive function project synchronization of Rössler hyperchaotic system with uncertain parameters," Physics Letters A, vol. 372, no. 20, pp. 3667-3671, 2008.

[12] H. Du, Q. Zeng, and C. Wang, "Function projective synchronization of different chaotic systems with uncertain parameters," Physics Letters A, vol. 372, no. 33, pp. 5402-5410, 2008.

[13] M. T. Yassen, "Adaptive control and synchronization of a modified Chua's circuit system," Applied Mathematics and Computation, vol. 135, no. 1, pp. 113-128, 2003.

[14] Z. Li, C. Han, and S. Shi, "Modification for synchronization of Rössler and Chen chaotic systems," Physics Letters A, vol. 301, no. 3-4, pp. 224-230, 2002.

[15] Y. Wang, Z.-H. Guan, and H. O. Wang, "Feedback and adaptive control for the synchronization of Chen system via a single variable," Physics Letters A, vol. 312, no. 1-2, pp. 34-40, 2003.

[16] J. Huang, "Adaptive synchronization between different hyperchaotic systems with fully uncertain parameters," Physics Letters A, vol. 372, no. 27-28, pp. 4799-4804, 2008. 
[17] D. Huang, "Stabilizing near-nonhyperbolic chaotic systems with applications," Physical Review Letters, vol. 93, no. 21, Article ID 214101, 2004.

[18] D. Huang, "Simple adaptive-feedback controller for identical chaos synchronization," Physical Review E, vol. 71, no. 3, Article ID 037203, pp. 1-4, 2005.

[19] D. Huang, "Adaptive-feedback control algorithm," Physical Review E, vol. 73, no. 6, article 066204, p. 8, 2006.

[20] Q. Jia, "Hyperchaos generated from the Lorenz chaotic system and its control," Physics Letters A, vol. 366, no. 3, pp. 217-222, 2007.

[21] X. Wang and M. Wang, "A hyperchaos generated from Lorenz system," Physica A, vol. 387, no. 14, pp. 3751-3758, 2008

[22] T. Gao, G. Chen, Z. Chen, and S. Cang, "The generation and circuit implementation of a new hyperchaos based upon Lorenz system," Physics Letters A, vol. 361, no. 1-2, pp. 78-86, 2007.

[23] Y. Li, W. K. S. Tang, and G. Chen, "Generating hyperchaos via state feedback control," International Journal of Bifurcation and Chaos in Applied Sciences and Engineering, vol. 15, no. 10, pp. 3367-3375, 2005.

[24] Z. Yan, "Controlling hyperchaos in the new hyperchaotic Chen system," Applied Mathematics and Computation, vol. 168, no. 2, pp. 1239-1250, 2005. 


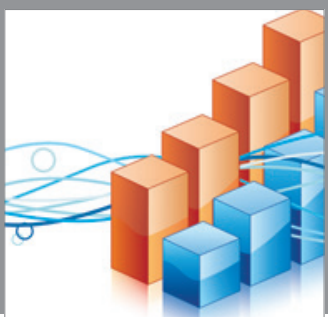

Advances in

Operations Research

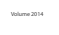

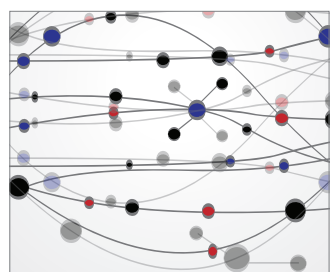

\section{The Scientific} World Journal
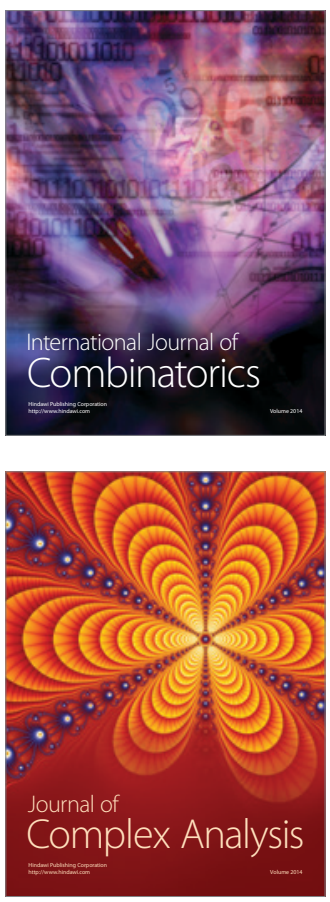

International Journal of

Mathematics and

Mathematical

Sciences
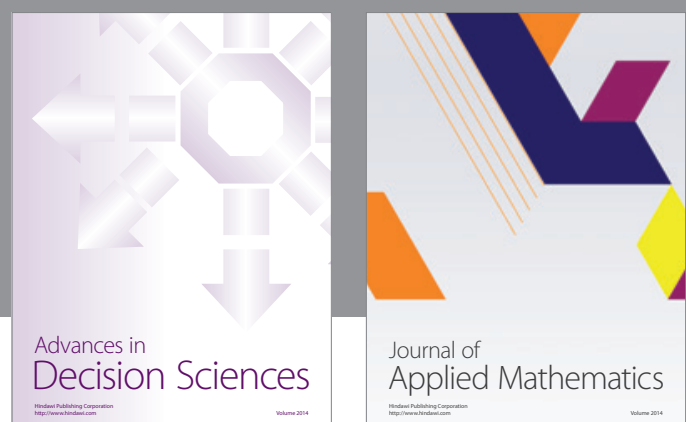

Journal of

Applied Mathematics
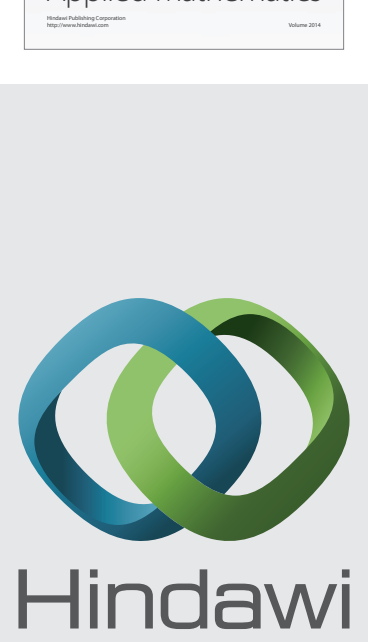

Submit your manuscripts at http://www.hindawi.com
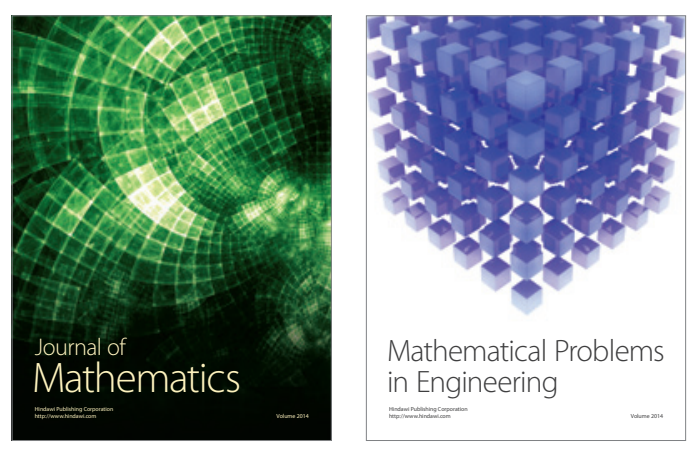

Mathematical Problems in Engineering
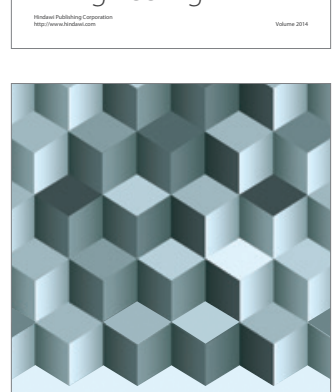

Journal of

Function Spaces
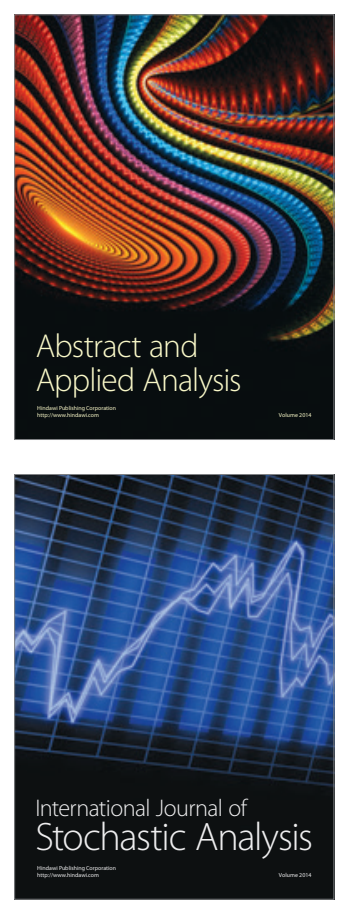

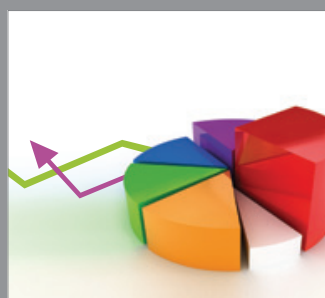

ournal of

Probability and Statistics

Promensencen
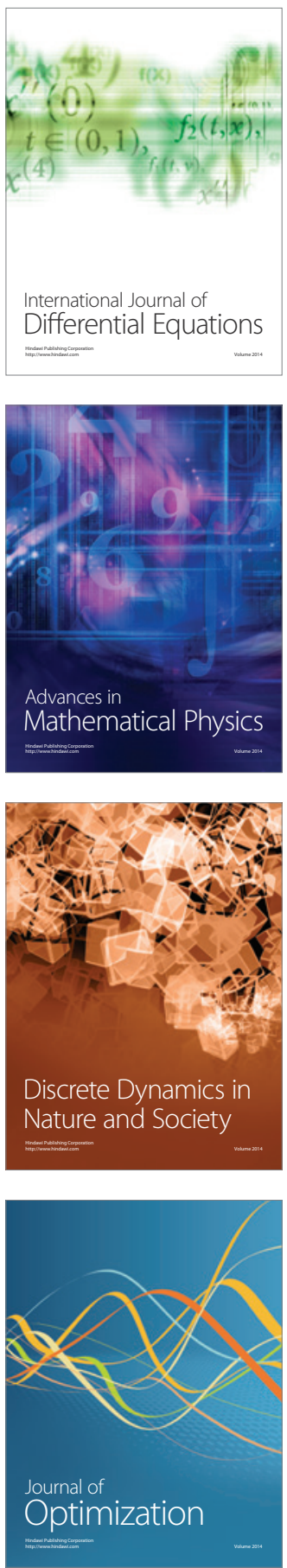